\title{
Boron Bromide as a Source Precursor for Low Energy Applications
}

\author{
R. Gwilliam \\ Surrey Ion Beam Centre \\ Advanced Technology Institute \\ School of Electronics and Physical Sciences \\ University of Surrey \\ Guildford, Surrey, UK. \\ r.gwilliam@surrey.ac.uk
}

\begin{abstract}
The use of boron tri-bromide as a source precursor for low energy boron implantation is studied in this work. Details of gas delivery system, beam purity and running performance have been investigated for a $75 \mathrm{keV}$ molecular implant to give an equivalent boron energy of $5 \mathrm{keV}$. The behavior of the $\mathrm{Br}$ during thermal processing is compared to that of $\mathbf{F}$ derived from a $\mathbf{B F}_{2}$ implant and is seen to be less mobile during post implant rapid thermal annealing with implications to gate oxide performance.
\end{abstract}

Keywords-boron bromide; shallow junctions; doping;

\section{INTRODUCTION}

The requirement for shallow junctions has seen the drive by equipment manufacturers to continually advance the lower energy operation window of implant tools over the last decade. This comes not without compromise, as the transport of high beam currents at low energies is beleaguered with problems caused by space charge issues resulting in beam blow up. Lower implant energies can be achieved with respectable currents however, if the majority of the transport of the beam is performed at higher energies with the final energy being as a result of a deceleration close to the target. This technique is however subject to energy contamination from ions becoming neutralized by a charge exchange process before entering the final deceleration field, resulting in dopant tails from the higher energy neutralized B. This can be particularly prevalent where photorisist out gassing may compromise the vacuum levels.

One technique that has been used for many years is to implant the halide $\mathrm{BF}_{2}^{+}$where, the energy is split between the $\mathrm{F}$ and the $\mathrm{B}$ resulting in a shallower $\mathrm{B}$ profile than would be achieved by a comparable $\mathrm{B}^{+}$implant at the same energy. As device dimensions have been reduced, $\mathrm{F}$, due to its mobility during thermal processing, has been identified as a cause of failure in the very thin gate oxides currently used. Previously, there have been several studies on the effects of halogens coimplanted with B [1-5] which have provided useful information on the implant defects and diffusion of $\mathrm{B}$ and the resulting electrical performance. In this work, $\mathrm{BBr}_{3}$ is investigated for its practicality for use, as an alternate precursor for machines unable to access the very low energies currently required for shallow junction formation and on the diffusion behavior of $\mathrm{Br}$ during rapid thermal annealing.

\section{EXPERIMENTAL}

\section{A. Source Precurser Delivery}

The $\mathrm{BBr}_{3}$, which is a liquid at room temperature, was attached to the ion source using a dedicated gas supply line attached to standard laboratory glass file. Delivery to the source was controlled with a precision needle valve operated remotely. A parallel Ar support gas was also available via the standard gas delivery system to both initiate source start up and to stabilize the plasma. An argon plasma was also run after closure of the $\mathrm{BBr}_{3}$ supply to clean the source prior shut down. The $\mathrm{BF}_{2}$ implants were performed using the standard mass flow controlled toxic gas delivery system with a $\mathrm{BF}_{3}$ precursor, again with a parallel Ar support gas.

\section{B. Implantation and Measurement}

4" $<100>$ P doped CZ n-type Si was implanted at an energy of $75 \mathrm{keV}$ to a dose of $2 \times 10^{14}$ ions $\mathrm{cm}^{-2}$ with comparable implants of $\mathrm{BF}_{2}^{+}$performed at an energy of $22 \mathrm{keV}$ into a second set of samples. All the implants were performed on a 200kV Danfysik DF1090 ion implanter which uses a CHORDIS ion source that was run in cold gas mode, details of which can be found in [6]. Molecular implants at these energies give an equivalent $\mathrm{B}$ implant energy of $\sim 5 \mathrm{keV}$ which has a projected range of $20 \mathrm{~nm}$ in silicon. Following implantation, samples were annealed at $1050^{\circ} \mathrm{C}$ for 10 seconds in an AG610 rapid thermal annealer under a flowing $\mathrm{N}_{2}$ ambient with a ramp rate of $50^{\circ} \mathrm{C} \mathrm{s}^{-1}$. SIMS analysis was performed on the asimplanted and the annealed samples to investigate the atomic profile of the $\mathrm{B}, \mathrm{Br}$ and $\mathrm{F}$. The analysis was performed on a Cameca $4 \mathrm{~F}$ using $\mathrm{O}_{2}^{+}$primary ion bombardment at $10 \mathrm{keV}$ with positive secondary ion detection to optimize the sensitivity to boron. Good sensitivity to fluorine may also be achieved under these conditions. $7.5 \mathrm{keV} \mathrm{Cs}^{+}$primary ion bombardment and negative secondary ion detection was used to optimize the

Funded by UK Engineering and Physical Sciences Research Council 
sensitivity to bromine. The depth scales were determined by measuring the sputter crater depths by interference microscopy with an accuracy of $10 \mathrm{~nm}$.

\section{RESULTS}

Fig. 1 shows the expected mass distribution of the $\mathrm{BBr}_{2}$

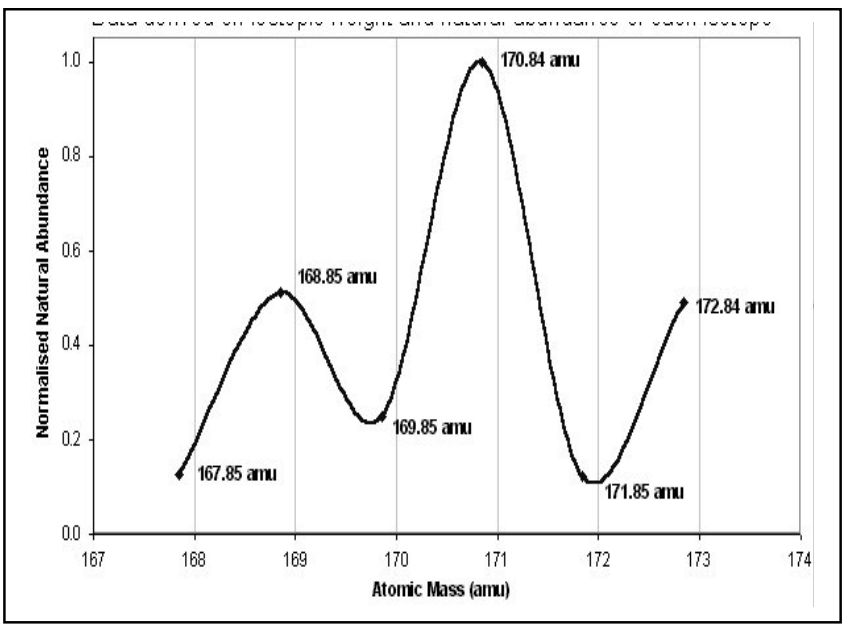

Figure 1 Predicted mass scan from natural isotope abundance

implant derived from the natural abundance of the isotopes. Fig. 2 shows a portion of the actual mass scan taken from the

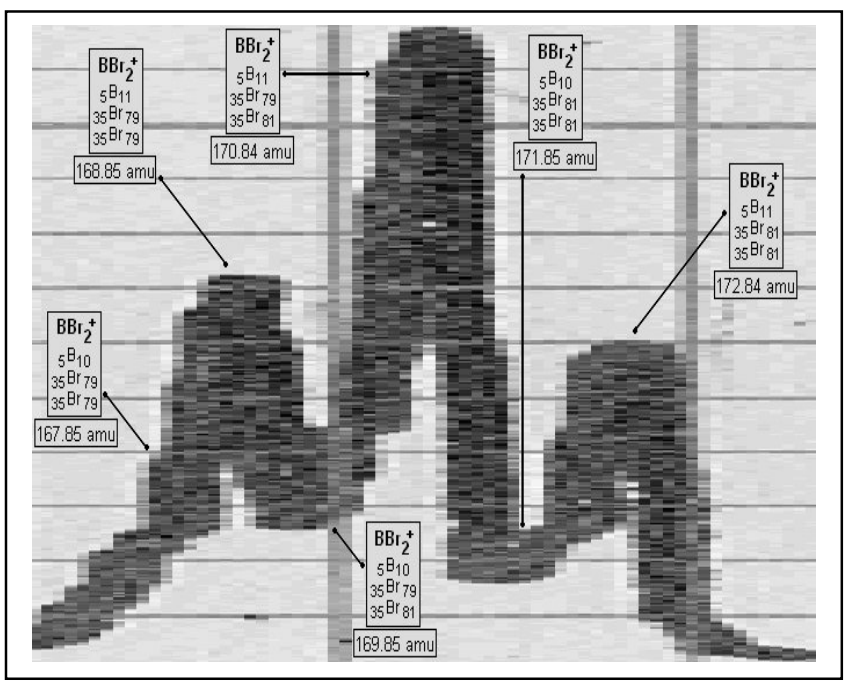

Figure 2. Actual isotope abundance for $\mathrm{BBr}_{2}$

machine relative to the desired ion species. The resolution of the mass scan is compromised as the mass resolving slits on the machine were set at a maximum for full beam transport. The overall shape of the scan is however as predicted. With the major peak being from the molecule constructed of $\mathrm{Br}_{79} \mathrm{Br}_{81} \mathrm{~B}_{11}{ }^{+}$isotopes which was the chosen implantation species The $\mathrm{BF}_{2}{ }^{+}$implants were performed using the major peak not described here. Fig. 3 shows the implanted $\mathrm{Br}$ profiles

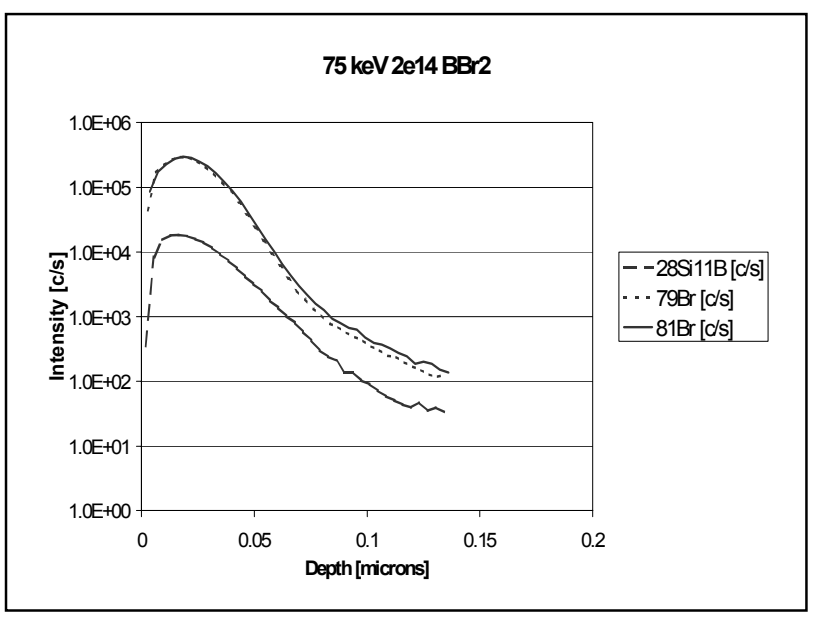

Figure 3 SIMS profile of the $\mathrm{B}$ and $\mathrm{Br}$ isotopes following implantation. The values are relative as no calibration standard was used

along with the $\mathrm{B}$ profile taken during the same SIMS measurement run. The $\mathrm{B}$ profile was derived from the ${ }^{28} \mathrm{Si}^{11} \mathrm{~B}$ ion obtained under the $\mathrm{Cs}^{+}$sputter conditions. This is not optimum for the measurement of $\mathrm{B}$, but gives a useful guide as to the relative positions of the $\mathrm{Br}$ and $\mathrm{B}$. As can be seen from

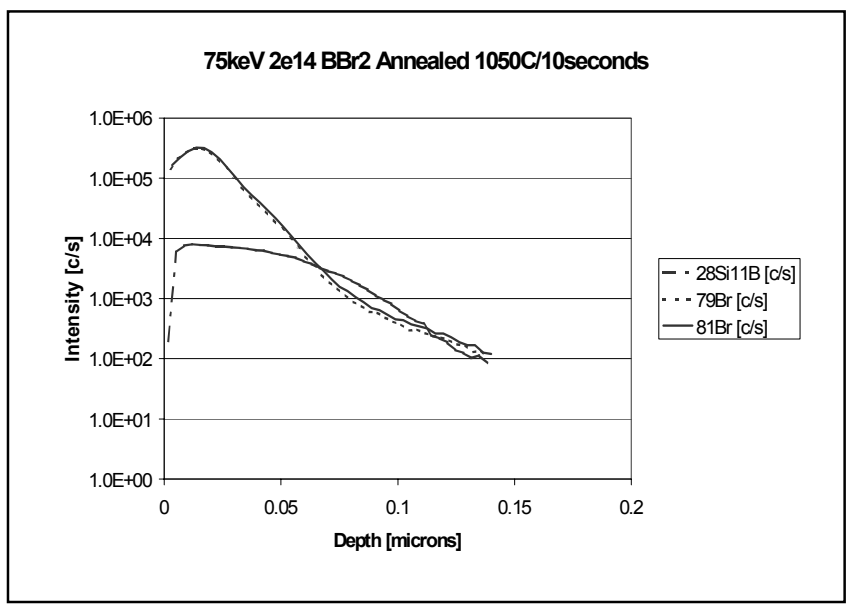

Figure 4 SIMS profile of the $\mathrm{B}$ and $\mathrm{Br}$ isotopes following implantation and annealing. Note $\mathrm{B}$ position relative to $\mathrm{Br}$

the measurement, the levels of ${ }^{19} \mathrm{Br}$ and ${ }^{81} \mathrm{Br}$ are very similar indicating that the ion species was primarily composed of ${ }^{79} \mathrm{Br}{ }^{81} \mathrm{Br}$ plus a boron isotope as expected.

Fig. 4 shows the profiles for the sample following the $1050^{\circ} \mathrm{C} 10$ second anneal. The Br profiles, while broader than the as implanted profiles, do not show significant redistribution compared to that observed for a comparable $\mathrm{BF}_{2}$ implant processed under the same annealing conditions as shown in Fig. 5 and 6 . The F signal is not quantified and is shown only as a count rate. As can be seen from the annealed sample when compared to the as implanted sample, the $\mathrm{F}$ is highly mobile and has redistributed decorating the damage giving a double hump profile along with diffusion to the surface. This is previously widely documented in the literature. 
Fig. 7 and Fig. 8 show the boron profiles for the as-

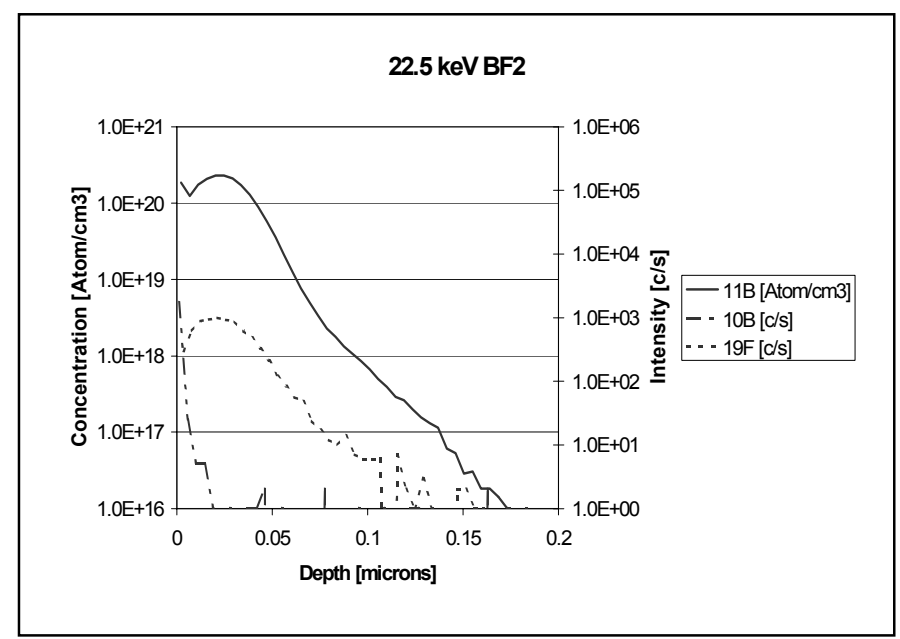

Figure 5 Boron and Fluorine distribution for as implanted $22.5 \mathrm{keV} \mathrm{BF} 2$ implant following implantation. Boron concentration is calibrated while fluorine is relative

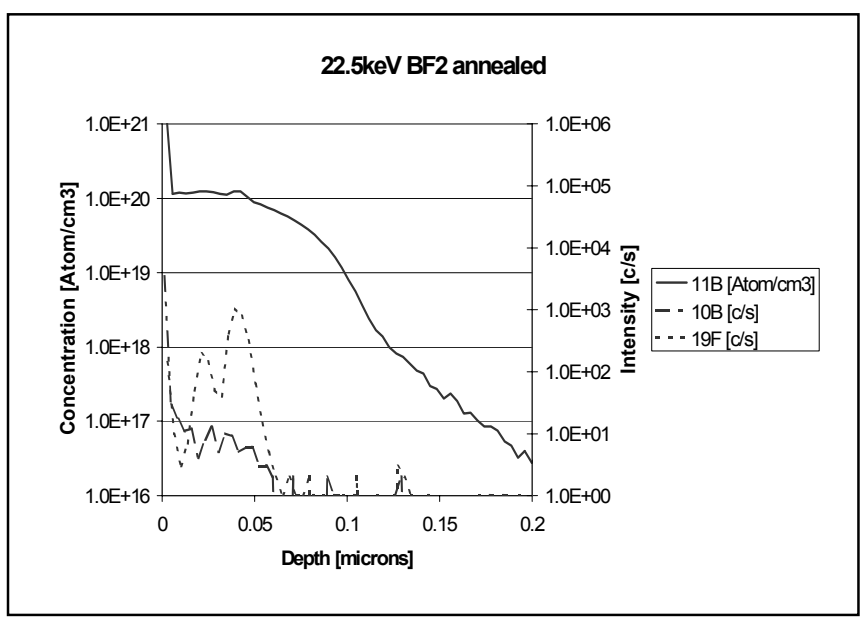

Figure 6 Boron and fluorine distribution following annealing at $1050^{\circ} \mathrm{C}$ for 10 seconds. Note redistribution of the fluorine to the surface, peak and end of range damage

implanted and annealed samples respectively with the profiles being taken using an $\mathrm{O}_{2}^{+}$primary ion beam and calibrated against a standard for absolute quantification. The as-implanted profile shape is consistent with that expected from a $5 \mathrm{keV} \mathrm{B}^{+}$ implant into damaged/amorphous material with little evidence of channeling and is certainly sharper than that observed for the $\mathrm{BF}_{2}$ implant as might be expected. Both ${ }^{10} \mathrm{~B}$ and ${ }^{11} \mathrm{~B}$ was profiled to ascertain the exact implanted isotopes for the $\mathrm{BBr}_{2}$ implant. As can be seen there are significant quantities of ${ }^{10} \mathrm{~B}$ present in the implant indicating that the implant consisted more than one molecular configuration.

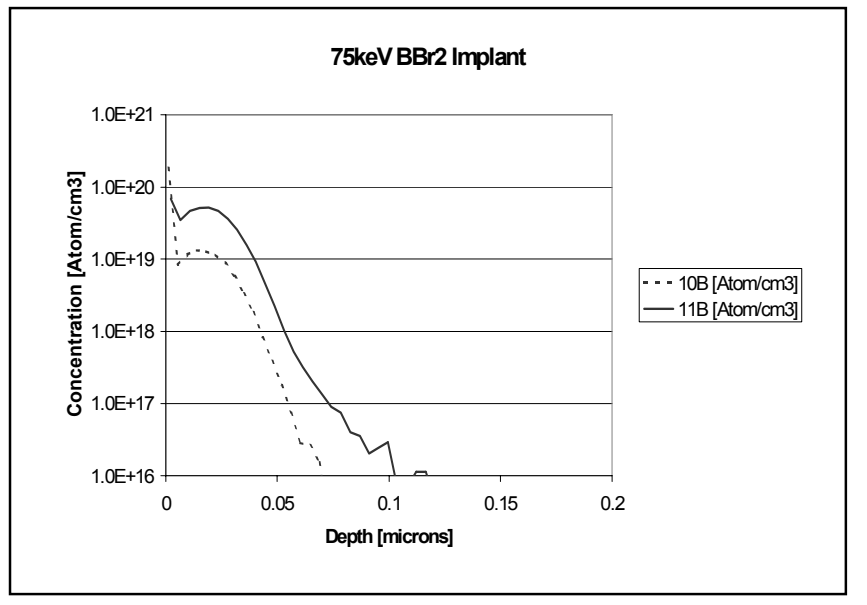

Figure7 Absolute boron concentration for $\mathrm{BBr}_{2}$ implantation after implantation

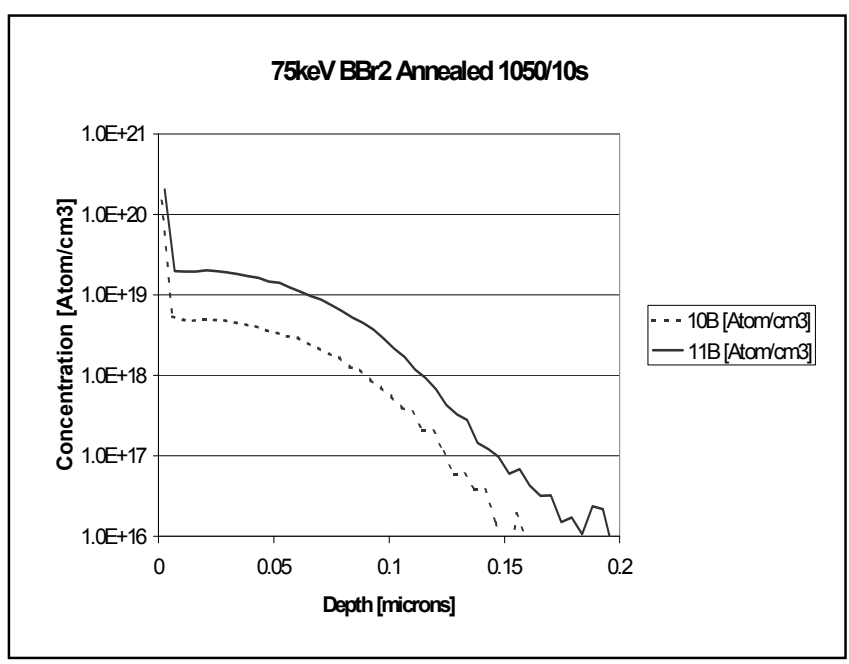

Figure 8 Absolute boron concentration for $\mathrm{BBr}_{2}$ implantation after implantation and annealing.. junction depth has extended some $100 \mathrm{~nm}$.

\section{DISCUSSION AND CONCLUSIONS}

$\mathrm{BBr}_{2}$ implants have been successfully performed on a Danfsik 1090 research implanter using a CHORDIS ion source with only minor modifications to the standard gas delivery system. There was ample vapor pressure at room temperature to enable running of the source without the need for heating of the precursor. However, as is often experienced with bromides and given the complexity of the CHORDIS ion source design, which has large internal surface areas, the precursor was found to give large memory effects. This manifests itself in wetting of the internal components from moisture absorption making future pumping of the source difficult following source exchange. This was still evident even after running an $\mathrm{Ar}$ plasma under high discharge conditions for up to 1 hour prior to source removal. This of course would seriously compromise its use in a production facility.

The boron profiles achieved were consistent with an implant of $5 \mathrm{keV} \mathrm{B}{ }^{+}$into disordered material with very little 
evidence of channeling and somewhat sharper than those achieved for a $\mathrm{BF}_{2}$ implant. This is to be expected given that the bromine has a significantly higher mass than the $\mathrm{F}$ and would introduce lattice damage at a much higher rate.

Interestingly, the $\mathrm{Br}$ has completely different diffusion behavior than the $\mathrm{F}$ with little evidence of major redistribution being observed unlike that seen for the $\mathrm{F}$ in the $\mathrm{BF}_{2}$ case. This in itself could prove useful when the deleterious effects of the $\mathrm{F}$ on oxides is considered, as there seems to be no migration to the surface and the $\mathrm{Br}$ will be less reactive than the $\mathrm{F}$.

As was seen from the SIMS data, significant ${ }^{10} \mathrm{~B}$ was also implanted with what was believed to be the ${ }^{79} \mathrm{Br}^{81} \mathrm{Br}^{11} \mathrm{~B}$ molecule. As the adjacent isotope to the ${ }^{79} \mathrm{Br}^{81} \mathrm{Br}^{11} \mathrm{~B}$ molecule is ${ }^{79} \mathrm{Br}^{81} \mathrm{Br}^{10} \mathrm{~B}$ this may have led to the contamination due to the resolving slits being at their most open. However, the mass resolution of the tool is such that we would expect an adjacent isotope to be two orders down under these running conditions. It may be that the contamination emanated for a combination of reasons such as $\mathrm{H}$ contamination possibly as a result of moisture gettered by the bromide and beam instabilities during the implant.

\section{REFERENCES}

[1] H. Muller, H. Ryssel and K. Schmid. J. Appl. Phs. 43, 20061972

[2] D.G. Beanland. Solid State Electronics 21, 537, 1978

[3] D.F. Downey, J.W. Chow, E. Ishida and K.S. Jones. Appl. Phys. Lett. 52,12631998

[4] L.S. Robertson et al. The effect of impurities on diffusion and activation of ion implanted boron in silicon. Mat. Res. Soc. Symp. Vol. 610. B5.8.1 2000

[5] S. Winston et al. Evaluation of the B activation and depth distribution using $\mathrm{BBr}_{2}$ implants. Presented IIT2002. To be published.

[6] . R. Keller, P. Spadtke and F. Nohmayer. Proceedings of International Ion Engineering Congress., Kyoto, Institute of Electrical Engineers of Japan, Tokyo, p. 251983 